\title{
Conquering the conquered: sternal tuberculosis osteomyelitis with hiv infection
}

\section{Letter to editor}

Tuberculosis is a disease caused by bacteria of the Mycobacterium tuberculosis complex and is one of the oldest diseases known. ${ }^{1}$ As per WHO report, in the year 2015 about 10.4 million new cases of tuberculosis were detected worldwide, of these 1.2 million cases were having infection by Human immunodeficiency virus (HIV). The burden of the co-infection is much more than thought as about $55 \%$ of notified tuberculosis patients had HIV infection. Along with the morbidity involved in the illness about 1.4 million deaths occurred of these 0.4 million were seen in HIV infected cases. India is one of the six countries which account for about $60 \%$ of all the cases. ${ }^{2}$ The clinical manifestation in tuberculosis (TB) may be pulmonary or extra pulmonary. In pulmonary tuberculosis the lungs are predominantly involved with or without involvement of pleura and lymph nodes. While in extra pulmonary tuberculosis most of the organs of the body can be involved like peritoneum, genitourinary tract, gastrointestinal, cardiac, bones, joints, meninges. Virtually all organs may be involved. In case of bones the weight bearing joints like the spine, hips and knees are more commonly affected.

In HIV infected individuals extra pulmonary tuberculosis is more common than pulmonary tuberculosis and hematological dissemination of the bacilli is one of the major reasons for this to occur. Also, it is seen that in these individuals the disease progresses over weeks rather than in months or years. The most common presentations being tuberculosis of lymph nodes, pleura, pericardium with or without dissemination. ${ }^{1}$ Tubercular involvement of the sternum in the form of osteomyelitis is rare. ${ }^{3}$ The presentation is in the form of indolent, painless course without constitutional symptoms like evening low grade rise of temperature, loss of appetite, weight loss, night sweats or sometimes with a more aggressive course causing painful bone destruction with constitutional symptoms. ${ }^{4}$ While the involvement of bone marrow in patients with HIV infection and presentation as osteomyelitis is uncommon. ${ }^{5}$ However, in case of sternum the superficial location of the wound brings it to notice of the patient. ${ }^{6}$

Wound on the anterior chest wall as seen in Sternal osteomyelitis is uncommon; review of literature reveals its occurrence only in few situations like in children following BCG vaccination and children with Thalassemia. Likewise in adults it is seen in uncontrolled diabetics, drug addicts with HIV infection and following sternotomy for cardiac surgery. ${ }^{7-12}$ Other than mycobacterium tuberculosis wound over sternum is reported in infection by staphylococcus aureus. ${ }^{13}$ Tubercular Sternal osteomyelitis is usually caused by reactivation of latent loci formed during hematogenous or lymphatic dissemination of primary tuberculosis. Studies by few authors have found extrasternal involvement presenting as bilateral pleural effusion or as involvement of other organs. ${ }^{6-16}$ As per WHO guidelines if an individual is diagnosed to have HIV infection then it is recommended to start screening for tuberculosis before starting antiretroviral therapy and also to continue screening at regular intervals and vice versa. The various screening and surveillance tools are focused towards symptoms of cough, night sweats and weight loss. ${ }^{17}$ Mantoux test
Volume I Issue 4 - 2017

\author{
Rupali Awale \\ Department of Laboratory Medicine, AllMs, India
}

Correspondence: Rupali Awale, Department of Laboratory Medicine, Ex-Senior Resident, AllMs, India, Email drawalerupali@gmail.com

Received: October 28, 2017| Published: November 27, 2017

has no value in coinfection as the skin test may be negative due to anergy. ${ }^{18}$ For investigating the patient culture of Mycobacterium tuberculosis is more sensitive as compared to smear microscopy and thus recommended in the diagnosis of tuberculosis in HIV infected case but the long time consumed in waiting for the culture report is a limitation, as the treatment needs to be started as soon as possible especially if the patient is immuno compromised and carries risk of progression to disseminated tuberculosis. ${ }^{19,20}$ Nucleic acid amplification testing provides specificity of diagnosis and is a rapid test, however the test requires expertise and is expensive so cannot be done at peripheral health centers'. ${ }^{18}$

The reliable option to this is Gene expert a TB specific automated cartridge based nucleic acid amplification assay which provides results within 100 minutes as well as reports sensitivity to Rifampicin. It can detect even the dead bacilli and give the report. ${ }^{18}$ Polymerase chain reaction can also be used in order to differentiate between other types of mycobacterium like mycobacterium intercellular, mycobacterium avium complex. While with the availability of gene expert it is not necessary. ${ }^{16}$ While testing by Interferon gamma release assay also known as QuantiFERON-TB Gold there is limitation of inability to differentiate between active and latent infection. ${ }^{19,20}$ Isolated case reports suggest estimation of vitamin D levels which were found to be low in their study but the rationale of conducting the test being unclear. ${ }^{5,21}$ As the patient has co-infection so treatment has to be started for both but the timing of initiation of treatment is important as drug interactions, drug metabolism and pharmacokinetics, patient tolerance to drugs and cost should be kept in mind. As these factors decide the compliance of patient and thus response to treatment.

It has been seen that early initiation of antiretroviral therapy (ART) within a month of initiation of anti tubercular treatment was associated with reduced mortality especially with CD4 counts as low as 50cells/ul. ${ }^{18}$ The standard recommended therapy consists of four drugs comprising of Rifampicin, Ionized, Pyrazinamide and Ethambutol followed by continuation phase comprising of Rifampicin and Ionized for four months. Here, the drug Rifampicin has a major role as it destroys the intracellular and slow growing bacilli thus higher cure rates without prolonging the duration of treatment. ${ }^{18-22}$ In India, NACO recommends antiretroviral regime consisting of Zidovudine or Stavudine along with Lamivudine and Efavirenz..$^{23}$ Here, it is also to be noted that Rifampicin an important drug in the treatment of tuberculosis is a cytochrome P450 enzyme inducer thereby increasing 
the metabolism of protease inhibitor and Non-nucleoside reverse transcriptase inhibitors (NNRTIs) thus causing therapeutic failure. ${ }^{24}$

Thus, to overcome this phenomenon the dose of Efavirenz is increased. ${ }^{18-25}$ Tuberculosis -Immune reconstitution inflammatory syndrome (TB-IRIS)] is sometimes seen after starting treatment because of high load of tuberculous bacilli and reactivation of T cells. ${ }^{26}$ While at the same time in patients of HIV infection active tuberculosis increases the rate of HIV virus replication and propagation because of failure of immunoregulation, proinflammatory cytokines and innate immunity signaling pathway activation thus requiring early initiation of treatment. ${ }^{26}$ The timing of initiation of ART and Anti tubercular therapy (ATT) is of debate irrespective of the CD4 count. ${ }^{19,20}$ If ART is started initially then there is reduction in early mortality as well as prevention of other opportunistic infections while if ATT is started initially then there are side effects like increased drug toxicity and drug interactions. ${ }^{18}$ Complications of Sternal tuberculosis include formation of fistula, spontaneous fracture of sternum or extension into pleural cavity or mediastinum,${ }^{14}$ thus making it necessary to give attention to the local treatment of the wound in addition to starting anti tubercular treatment. If the wound is big then good results have been seen with granulation tissue debridement followed by curettage of bony lesion and partial resection with reconstruction. ${ }^{6-10}$ To conclude, a high index of suspicion clubbed with investigation like biopsy and microbiological testing can aid in the prompt diagnosis at the earliest.

\section{Acknowledgements}

None.

\section{Conflict of interest}

The author declares no conflict of interest.

\section{References}

1. Raviglione MC. Tuberculosis. In: Kasper D, Fauci A, et al, editors. Harrison's Principles of Internal Medicine, 19e New York, USA; 2014. p. $482-490$.

2. World Health Organization. Global tuberculosis report 2016, Switzerland; p. $1-5$.

3. Sharma S, Juneja M, Garg A. Primary tubercular osteomyelitis of the sternum. Indian J Pediatr. 2005;72(8):709-710.

4. Jain A, Chawla S, Kumar M. Sternal tuberculous osteomyelitis: a series of four cases. Int J community Med Public Health. 2015;2(3):288-292.

5. Shafer RW, Kim DS, Weiss JP, et al. Extrapulmonary tuberculosis in patients with human immunodeficiency virus infection. Medicine (Baltimore). 1991;70(6):384-397.

6. Thakker T, Prabhakar MM, Patel DA. Tubercular osteomyelitis of sternum. Ind J Orthop. 2005;39(3):179-181.

7. Kato Y, Horikawa Y, Nishimura Y, et al. Sternal tuberculosis in a 9-monthold infant after BCG vaccination. Acta Paediatr. 2000;89(12):14951497.

8. Corrales IF, Cortes JA, Mesa ML, et al. Sternal osteomyelitis and scrofuloderma due to BCG vaccination. Biomedica. 2003;23(2):202207.
9. Kataria SP, Avasthi R. Sternal tuberculosis in combination with thalassemia. J Assoc Physicians India. 1993;41(7):472.

10. Saifudheen k, Anoop TM, Mini PN, et al. Primary tubercular osteomyelitis of the sternum. International Journal of Infectious Diseases. 2010;14(2):164e-166e.

11. Martors JA, Olm M, Miro JM, et al. Chondrocostal and chondrosternal tuberculosis in 2 heroin addicts infected with human immunodeficiency virus. Med Clin [Barc]. 1989;93(12):467-470.

12. Yew WW, Kwan SY, Ma WK, et al. Single daily dose ofloxacin monotherapy for Mycobacterium fortuitum sternotomy infection. Chest. 1989;96(5):1150-1152.

13. Vasa M, Ohikhuare C, Brickner L. Primary sternal tuberculosis osteomyelitis: A case report and discussion. Can J Infect Dis Med Microbiol. 2009;20(4):181e-184e.

14. Sharma SK, Mohan A. Extrapulmonary tuberculosis. Indian J Med Res. 2004;120(4):316-353.

15. Tuli SM, Sinha GP. Skeletal tuberculosis "Unsual" Lesions. Ind J Orthop. 1969;3(1):5-18.

16. Narang M, Dwivedi A, Narang S, et al. Sternal Tuberculosis: An Uncommon Presentation. J Case Rep. 2011;1:9-11.

17. Geneva: World Health Organization. Interim policy on collaborative TB/ HIV activities. 2004.

18. Padmapriyadarsini C, Narendran G, Swaminathan S. Diagnosis \& treatment of tuberculosis in HIV co-infected patients. Indian J Med Res. 2011;134(6):850-865.

19. Antiretroviral therapy for HIV infection in adults and adolescents World Health organization.

20. World Health Organization. Strategic and Technical Advisory Group (STAG-TB). Report of the $10^{\text {th }}$ Meeting. 2010.

21. Aseel Hegazi, Bojana Dragovic. Tuberculous osteomyelitis of the sternum in a patient with well controlled HIV infection and undetectable vitamin D levels. HIV \& AIDS Review. 2012;11(3):71-72.

22. Korenromp EL, Scano F, Williams BG, et al. Effect of human immunodeficiency virus infection on recurrences of tuberculosis after rifampin-based treatment: an analytical review. Clin Infect Dis. 2003;37(1):101-112.

23. World Health Organization. Improving the diagnosis and treatment of smear-negative pulmonary and extrapulmonary tuberculosis among adults and adolescents: recommendations for HIV-prevalent and resource-constrained settings. Switzerland; 2007. p. 1-44.

24. de Jong BC, Israelski DM, Corbett EL, et al. Clinical management of tuberculosis in the context of HIV infection. Annu Rev Med. 2004;55:283-301.

25. Manosuthi W, Sungkanuparph S, Thakkinstian A, et al. Efavirenz levels and 24-week efficacy in HIV-infected patients with tuberculosis receiving highly active antiretroviral therapy and rifampicin. AIDS. 2005;19(14):1481-1486.

26. Bell LCK, Noursadeghi M. Pathogenesis of HIV-1 and Mycobacterium tuberculosis co-infection. Nat Rev Microbiol. 2018;16(2):80-90. 